Meta

Journal des traducteurs

Translators' Journal

\title{
Quelques organismes travaillant en terminologie scientifique, technique ou professionnelle pour les langues espagnole et portugaise
}

\section{Daniel Prado}

Volume 35, numéro 3, septembre 1990

La traduction dans le monde hispanolusophone

URI : https://id.erudit.org/iderudit/003864ar

DOI : https://doi.org/10.7202/003864ar

Aller au sommaire du numéro

Éditeur(s)

Les Presses de l'Université de Montréal

ISSN

0026-0452 (imprimé)

1492-1421 (numérique)

Découvrir la revue

Citer cet article

Prado, D. (1990). Quelques organismes travaillant en terminologie scientifique, technique ou professionnelle pour les langues espagnole et portugaise. Meta, 35(3), 561-570. https://doi.org/10.7202/003864ar d'utilisation que vous pouvez consulter en ligne. 


\title{
QUELQUES ORGANISMES TRAVAILLANT EN TERMINOLOGIE SCIENTIFIQUE, TECHNIQUE OU PROFESSIONNELLE POUR LES LANGUES ESPAGNOLE ET PORTUGAISE
}

\author{
DANIEL PRADO \\ Union latine, Paris, France
}

\section{INTRODUCTION}

Le présent article prétend décrire certaines activités terminologiques telles que la collecte, la recherche, l'enseignement, l'utilisation et la planification terminologique réalisées pour les langues espagnole et portugaise.

Ces langues, qui souffrent d'un retard considérable en matière de vocabulaires scientifiques et techniques auquel aucune politique de l'État ne cherche à remédier' ${ }^{1}$, constituent, paradoxalement, des pôles d'attraction tant pour des organisations internationales que pour des entreprises vouées à ce qu'on appelle les industries de la langue. En effet, l'évolution démographique ${ }^{2}$, la présence de plus en plus accentuée dans les organisations internationales ${ }^{3}$ et d'autres facteurs mineurs mais néanmoins significatifs ${ }^{4}$ font que ces langues prennent chaque jour plus d'importance, en particulier dans le monde de la documentation technique et professionnelle.

C'est ainsi que certains éditeurs européens non-ibériques, des organisations internationales comme la CÉE ou des bureaux qui ont des systèmes de traduction automatique comme Systran ou Siemens, développent par rapport à l'espagnol et au portugais une activité terminologique parfois plus importante que celle qui est réalisée dans les pays ibéro-américains eux-mêmes 5 .

Dans ces pays, les activités concernant le domaine en question sont, sans aucun doute, prolifiques mais souffrent d'un manque d'initiatives émanant de l'État (elles sont mal diffusées, atomisées et, fondamentalement, inorganisées) ${ }^{6}$. C'est pourquoi des institutions internationales comme Infoterm et l'Union latine mènent certaines actions de coordination et de sensibilisation auprès des autorités compétentes afin que celles-ci dotent les organismes nationaux qui travaillent dans ce domaine de plus amples moyens financiers et logistiques.

\section{INSTITUTIONS LIÉES À LA TERMINOLOGIE EN ESPAGNOL ET EN PORTUGAIS}

\section{A. CLASSIFICATION RETENUE}

Établir une liste des institutions travaillant avec la terminologie espagnole ou portugaise signifie d'emblée proposer une classification à la fois en ce qui concerne le type de lien qui relie l'institution et la terminologie ${ }^{7}$ et la destination éventuelle du travail terminologique réalisé ${ }^{8}$. Une division complémentaire s'impose par ailleurs: l'origine de l'institution.

C'est ainsi que, pour des raisons pratiques, nous répartirons les institutions comme suit (cf. tableau annexe, point III) : 
- institutions ibéro-américaines: implantées en Europe ou en Amérique hispanophone et lusophone;

- institutions internationales: s'occupant de la terminologie espagnole ou portugaise parmi d'autres ;

- institutions non-ibéro-américaines: implantées hors de l'Europe ou de l'Amérique hispanophone et lusophone, mais s'intéressant entre autres à ces langues.

À l'intérieur de chaque chapitre nous indiquerons le type de relation existant entre l'institution et la terminologie ainsi que la destination des travaux terminologiques de la première. Le tableau synoptique du point III présente le résumé de ce qui est expliqué plus loin, tout en gardant la même classification et en faisant figurer le type d'activité et la destination des travaux terminologiques.

\section{B. INSTITUTIONS IBÉRO-AMÉRICAINES}

Parmi les institutions situées en Amérique hispanophone et lusophone ou dans la péninsule ibérique, nous devons tout particulièrement signaler les suivantes:

\section{Pour la langue espagnole :}

Asociación Española de Traductores e Intérpretes (APETI)

Assure la formation et l'information terminologique de ses membres.

Centro de Investigaciones para Industrias del Lenguaje (CIPIL) à Buenos Aires

Axé en priorité sur le développement de la linguistique informatique, ce centre projette de développer des activités en terminologie et lexicographie telles: «des banques et bases de données terminologiques, documentaires», «des vocabulaires, dictionnaires et thésaurus mono- et plurilingues», «des systèmes d'indexation assistée par ordinateur», «des systèmes de traduction assistée par ordinateur», etc.

\section{Centro de Información Científica y Humanística (CICH) de l'Universidad Autónoma à} Mexico

Réalise les projets suivants : traduction des termes de base en science et technologie, traduction en espagnol du thésaurus de l'ASFIS, normalisation des descriptions de la base de données CIASE et de l'Indice periódica, préparation d'une base de données comportant les sigles et acronymes de l'Amérique latine.

\section{Colégio de México de la ville de Mexico}

a) Le département P.F.T. (Programme de formation de traducteurs), outre qu'il a assuré la formation en terminologie (destinée aussi bien à des étudiants qu'à la formation de terminologues spécialisés), a également élaboré des glossaires sur les nouvelles énergies et les énergies renouvelables entre autres.

Suite à un accord avec Eurodicautom, une banque terminologique est en cours de constitution.

b) Le département du Dictionnaire de l'espagnol réalise le dictionnaire de l'espagnol parlé au Mexique à partir d'un analyseur de fréquences. Ce département à réalisé en outre, pour l'Union latine, la version mexicaine de l'inventaire des ressources terminologiques de l'espagnol.

\section{Colegio de Traductores Públicos à Buenos Aires}

Assure la formation en terminologie et en linguistique informatique des traducteurs membres par le biais de séminaires et de cours.

Crée de la terminologie sur la base d'accords passés avec TEAM et Eurodicautom. 
Propose à ses membres un travail de recherche et d'expérimentation en matière de traduction automatique.

\section{Comunicación y Lingüística à Madrid}

Il s'agit d'une société commerciale qui travaille sur l'espagnol et sur le portugais, entre autres. Actuellement, elle se consacre au projet PREDICAT, qui comporte la formation et la collecte terminologique, la création de banques de données terminologiques et documentaires, l'édition de dictionnaires et l'élaboration de logiciels spécialisés en linguistique.

En outre, cette société procède à la collecte terminologique et assure une formation générale en documentation.

\section{Equipo de Investigación Terminológica (EIT) de la Pontificia Universidad Católica de} Chile de Santiago au Chili

Projette de constituer une banque de données terminologique à la suite d'une étude de faisabilité (Faisabilité d' une banque terminologique de la PUC au Chili).

Instituto Caro y Cuervo en Colombie

Réalise le Diccionario de Construcción y Régimen de la langue espagnole.

Instituto Venezolano del Petróleo (INTEVEP)

A constitué un glossaire des Procédés thermiques de l'extraction du pétrole et réalise actuellement un thésaurus sur l'industrie pétrolière.

HispanoTerm à Madrid

Institution pionnière dans la recherche terminologique : a réalisé la traduction de quelques ouvrages théoriques et a établi certaines bases importantes pour la recherche. Actuellement ne réalise aucune activité.

\section{ICFES / SIDES}

Se consacrant tout spécialement à l'information de l'enseignement supérieur, cette institution réalise le projet LEMB (liste des matières destinées aux bibliothèques).

INST. DE LITERATURA Y LINGUISTICA de Cuba

Sur le point de créer une unité terminologique.

Ministerio de Industria y Energía en Espagne

A réalisé un glossaire multilingue des termes utilisés dans le domaine de la qualité industrielle.

Real Academia de Ciencias Exactas, Físicas y Naturales en Espagne

A élaboré le Vocabulaire scientifique et technique dont la deuxième édition est en cours de réalisation (30 000 termes).

TermCat à Barcelone

Quoique son activité soit orientée spécifiquement vers la langue catalane, cet institut réalise un travail de haut niveau sur la langue espagnole. Institution de recherche, de compilation, de consultation et de formation terminologique qui a sous sa responsabilité la normalisation du catalan. Sa banque de terminologie multilingue - l'espagnol y figure parmi d'autres langues - constitue une référence exemplaire en la matière.

TermEsp à Madrid

Groupe émanant de l'ICYT (Instituto de Información y Documentación Científica) du CSIC (Consejo Superior de Investigaciones Científicas de España), TermEsp s'occupe de 
la collecte et de la traduction de termes, de la formation en terminologie et de la création de thésaurus et de vocabulaires parmi d'autres activités liées à la terminologie et à l'information scientifique et technique en général.

La CÉE l'a chargé de la traduction en espagnol des termes relatifs à des secteurs techniques et professionnels. On peut citer parmi ses travaux: le glossaire des termes métallurgiques, le thésaurus de la Science pharmacologique et des termes métallurgiques (VE), le dictionnaire de la FID (VE) et des sciences de l'alimentation et des aliments, le catalogue des ressources terminologiques en langue espagnole, etc.

Tribunal de Cuentas en Espagne

A publié le glossaire espagnol-chinois de la terminologie de la Cour des Comptes.

Universidad Simón Bolívar à Caracas

a) Groupe de recherche terminologique (GIT) : institution à caractère technologique qui assure la formation en terminologie de l'université. A réalisé un glossaire technique pour le métro de Caracas et trouvé les équivalences espagnoles du Thésaurus sur l'économie et l'énergie. A collaboré avec des sociétés commerciales en vue de l'élaboration de glossaires. A organisé le Ier Séminaire vénézuelien de terminologie et le Ier Symposium latino-américain de terminologie au cours duquel a été créé le «Red Iberoamericana de Terminología» (Réseau ibero-americain de terminologie).

b) Le groupe de la banque de terminologie de l'université Simón Bolívar (BTUSB) se consacre à la mise en route de banques terminologiques multilingues basées sur le système des Nations Unies et qui travaillent sur un ordinateur personnel. Cette banque est déjà opérationnelle, elle se trouve néanmoins encore en cours de développement.

UZEI-EuskalTerm à San Sébastián (Espagne)

Comme pour le Termcat, les activités terminologiques de cette institution ne concernent qu'indirectement l'espagnol, étant donné que son objet d'étude est avant tout la langue basque.

Possède une banque de terminologie multilingue - où figure l'espagnol - qui peut être consultée en ligne. A réalisé une grande quantité de dictionnaires et de glossaires, en général quadrilingues: basque, espagnol, français et anglais.

\section{Pour la langue portugaise :}

Centro de Linguística da Universidade de Lisboa

(Institut appartenant à l'Instituto Nacional de Investigação Científica-INIC).

Réalise, entre autres, un dictionnaire électronique et un lexique-grammaire de la langue portugaise, constitue actuellement un corpus de référence du portugais européen contemporain en vue d'études linguistiques.

Centro de Tecnologia Educativa de Projecto Universidade Aberta au Portugal Élabore un glossaire de l'audiovisuel portugais-français-anglais.

Centro português de Terminologia : Academia das Ciências de Portugal et ICALP

L'Académie des sciences du Portugal comporte également le secteur des sciences humaines et joue ainsi, en outre, le rôle d'Académie de la langue. Élabore à l'heure actuelle un dictionnaire de la langue. L'Académie et l'ICALP (Institut de culture et langue portugaises) ont récemment créé un Centre de terminologie. Les principaux objectifs de ce centre sont: «coordonner l'activité de la production terminologique au Portugal, mener à bien l'unification terminologique avec le Brésil et les pays africains lusophones, établir des échanges avec les organismes internationaux similaires, collaborer à la consti- 
tution de banques terminologiques, participer à l'élaboration de l'inventaire des lacunes terminologiques de la langue portugaise». Parmi ses premières réalisations figure la création de sept commissions de terminologie (deux de ces commissions ont été créées à l'initiative de l'Union latine).

\section{Comissão Nacional de Língua Portuguesa}

Créée en vue de conseiller le Premier ministre en matière de politique linguistique portugaise, elle compte parmi ses attributions la terminologie scientifique et technique.

Departamento de inglês da Universidade de Ouro Preto au Brésil

Procède à la constitution d'une banque de données du langage des sciences minières et géologiques, de la métallurgie et du génie civil.

\section{EMBRATEL-DPD}

Développe des systèmes de traitement du langage naturel, surtout pour la consultation de base de données, pour lesquels on crée et utilise des dictionnaires électroniques.

\section{FFLCH - Universidade de São Paulo}

Le Département d'études roumaines élabore un dictionnaire roumain-portugais. Il existe un département de lexicographie automatisée.

IBICT à Brasilia

L'Institut brésilien d'information scientifique et technique a pour principale tâche de coordonner au Brésil des activités en matière d'information et de documentation et vient de créer un groupe de travail terminologique qui s'occupera, entre autres, de l'inventaire des ressources terminologiques de la langue portugaise.

\section{INCITE}

Travaille sur des thésaurus. A réalisé l'inventaire des sources et des ressources terminologiques au Portugal.

\section{Instituto de Investigação Científica Tropical}

Participe à plusieurs commissions techniques de normalisation et de contrôle de la qualité et réalise la version portugaise du thésaurus AGROVOL, un glossaire de termes de l'industrie du pétrole et un glossaire et une bibliographie micro-paléontologique.

\section{LEXIKON à Rio de Janeiro}

Cette société privée réalise des logiciels de correction orthographique, grammaticale et de style. Elle est spécialisée dans la linguistique informatique.

\section{Núcleo de Estudos de Linguística Contrastiva da Universidade Nova de Lisboa}

Développe un projet intitulé «Néologie du portugais contemporain» qui comporte deux parties : un manuel de création néologique et le développement d'une banque de néologismes.

\section{Universidade Estadual Paulista «Julio de Mesquita Filho» (UNESP)}

L'Institut des lettres, des sciences sociales et de l'éducation présente un projet d'élaboration d'un dictionnaire standard du portugais contemporain à partir de la constitution d'un dictionnaire des fréquences du portugais contemporain et d'accord de textes.

\section{INSTITUTIONS INTERNATIONALES}

Infoterm. Siège : Vienne

Organisation mondiale de terminologie créée par l'UNESCO. Sa mission principale est d'orienter et de coordonner les activités terminologiques au niveau international. A édité 
toute une série d'ouvrages de références, de catalogues, de répertoires sur les dictionnaires, de thèses et de manuels. A créé un réseau international de terminologie (TermNet) qui publie un bulletin (TermNet News). Actuellement, elle se consacre à la constitution d'une base de données bibliographiques et factographiques et prépare un projet destiné à rendre plus facile le transfert des connaissances en matière de terminologie. A organisé également plusieurs rencontres internationales.

Bureaux de terminologie des Institutions de la Communauté économique européenne, Luxembourg, Bruxelles, Strasbourg

Le Parlement, la Cour de Justice et les Commissions de la CÉE réalisent une activité terminologique sans égale au bénéfice des langues espagnole et portugaise, parmi les langues officielles ( 9 au total). La banque de terminologie Eurodicautom contient environ 150000 termes espagnols et 100000 termes portugais, termes contenus dans les nombreux glossaires édités par le Service des publications.

\section{Organización Panamericana de la Salud}

Possède un système de traduction automatique pour les langues espagnole et portugaise.

\section{Organismes de la sphère des Nations Unies}

ONU, UNESCO, OACI, OMS, FAO, OIT, UIT, etc. Ces organismes ont constitué des groupes terminologiques dont la fonction première est de fournir les outils nécessaires aux services internes de traduction. Les glossaires et les vocabulaires qu'ils élaborent ont, dans la plupart des cas, une répercussion importante à l'extérieur. L'espagnol est la langue officielle dans la plupart de ces institutions.

Deuxième programme de l'Union latine. Siège: Saint-Domingue

Développe des activités de planification, de collecte, de recherche, d'information et joue le rôle d'intermédiaire en ce qui concerne les langues espagnole et portugaise, entre autres.

Parmi ses activités figurent: l'inventaire des ressources terminologiques; l'inventaire des lacunes terminologiques; la collecte des vocabulaires de l'intelligence artificielle, de l'aviation civile, de l'informatique, etc.; les manuels de créations néologiques; les enquêtes sur l'enseignement; la recherche et l'application de la linguistique informatique; l'organisation d'expositions et de conférences sur la terminologie; la traduction; l'édition de dictionnaires et la linguistique informatique; l'octroi de bourses et de subventions pour la formation en terminologie et en linguistique informatique. La publication du bulletin d'information et de liaison Terminomètre.

\section{Union des associations techniques internationales (UATI)}

Est dotée d'un programme spécifique à la terminologie. Participe à l'élaboration ou élabore des dictionnaires techniques et possède une banque de terminologie. L'espagnol y fait l'objet de certains travaux.

\section{INSTITUTIONS NON-IBÉRO-AMÉRICAINES}

\section{Agence linguistique européenne}

Société privée qui effectue des études linguistiques et des travaux de collecte terminologique espagnole et portugaise destinés à la CEE.

\section{Bureau de traductions du secrétariat d'État du gouvernement du Canada}

Traditionnellement, ce bureau s'est toujours consacré au binôme anglais-français, mais depuis quelques années, il élabore également des glossaires dans d'autres langues, notamment en espagnol. 


\section{CENTRE JACOUES-AMYOT}

Association française qui se consacre à l'échange et à la formation terminologique.

Administre des cours de terminologie et de traduction en Argentine et au Brésil.

HERIOT-WATT UNIVERSITY - DEPT. LANGUAGES

Le Département espagnol de cette université anglaise a créé une banque de néologismes espagnols.

\section{GÖTEBORGS UNIVERSITET}

Cette université a créé une banque de termes parus dans la presse espagnole au cours de l'année 1977 pour servir de base à des études.

Nous signalons ci-dessous les systèmes de traduction ou les banques de terminologie utilisant l'espagnol ou le portugais.

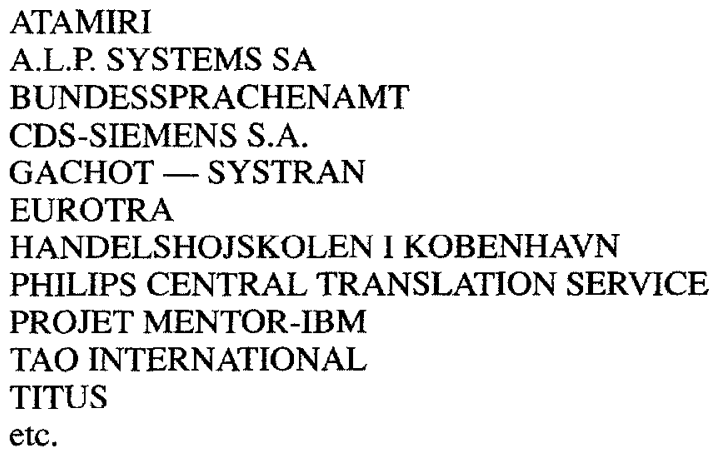

ATAMIRI

A.L.P. SYSTEMS SA

BUNDESSPRACHENAMT

CDS-SIEMENS S.A.

GACHOT — SYSTRAN

EUROTRA

HANDELSHOJSKOLEN I KOBENHAVN

PHILIPS CENTRAL TRANSLATION SERVICE

PROJET MENTOR-IBM

TAO INTERNATIONAL

TITUS

etc.

\section{TABLEAU SYNOPTIQUE}

\section{A) NOTICE EXPLICATIVE}

Le tableau synoptique qui suit présente de façon abrégée (et souvent approximative) certaines activités développées en matière de terminologie pour les langues espagnole et portugaise.

On y retrouve les institutions auxquelles on a fait référence dans l'article ainsi que quelques autres dont le rapport avec les activités en question est périphérique.

\section{a) INSTITUTION, SOCIÉTÉ OU ORGANISME}

Le tableau est dressé alphabétiquement par institution. On y indique le pays où se trouve chaque organisme (le siège pour les organisations internationales), et ce afin d'éviter les problèmes d'homonymie.

On a également séparé les activités appartenant à deux secteurs différents d'un même organisme (comme le Colegio de México ou l’Université Simón-Bolívar).

\section{b) ACTIVITÉ DÉVELOPPÉE EN MATIÈRE DE TERMINOLOGIE}

À chaque fois qu'il s'agit d'une institution développant (prioritairement ou partiellement) une activité en rapport avec la terminologie, on l'a indiqué par une croix.

Des renseignements qualitatifs ou quantitatifs n'ont pas été ajoutés en raison du manque d'espace. Il est évident que l'utilisation de la terminologie chez Embratel, au Brésil, est de loin inférieure à celle de la Communauté européenne et que le rôle de la collecte terminologique entreprise par AENOR est secondaire si l'on tient compte de son activité de normalisation. 
1) NORMALISATION (OU STANDARDISATION). Organismes dont les recommandations ont valeur de loi ou régissent de facto l'acceptation ou le refus de néologismes.

2) ENSEIGNEMENT. Institutions fournissant de façon permanente ou temporaire une formation en terminologie, soit comme discipline principale, soit comme discipline faisant partie d'un cursus.

3) COLLECTE. Institutions procédant à la collecte de termes de façon systématique ou occasionnelle.

4) UTILISATION. Institutions que leurs activités ayant trait à la traduction (CÉE) ou utilisant des systèmes de traduction automatique (SYSTRAN) entraînent à faire des recherches terminologiques pour le compte d'organismes de l'extérieur.

5) DIFFUSION. Institutions élaborant des glossaires, dictionnaires ou ouvrages terminologiques de diffusion générale ou qui proposent des services de banque de données.

6) PLANIFICATION. Organismes qui réalisent des activités terminologiques au niveau national ou international sur la base d'une politique terminologique ou linguistique.

Avertissement: pour les raisons citées ci-dessus - accès difficile à l'information -, les auteurs ont, très probablement, omis de citer dans cet article les activités d'autres institutions qui ont à leur actif, autant, si ce n'est davantage de tâches que celles qui sont présentées dans cet article.

Par ailleurs, les institutions auxquelles il est fait référence ici réalisent en matière de terminologie d'autres activités qui nous sont inconnues. Dans les deux cas, nous présentons toutes nos excuses à ces institutions et nous les invitons à nous envoyer toute information concernant leurs nouvelles activités.

\section{NOTES}

1. Le français, le russe, l'allemand, l'arabe jouissent d'un important soutien officiel en ce domaine.

2. Environ 500 millions.

3. L'espagnol est la langue officielle de la plupart des organisations mondiales et le portugais l'est également dans plus d'une dizaine d'organisations internationales.

4. Importante participation du Brésil et de l'Espagne au marché mondial, augmentation du pourcentage de locuteurs en espagnol aux États-Unis, etc.

5. Nous utiliserons le terme Ibéro-Amérique pour désigner l'ensemble des pays européens et américains qui ont pour langue officielle l'espagnol ou le portugais.

6. Le Portugal commence cependant à se doter d'une politique linguistique au niveau de l'État. Cette politique s'est récemment concrétisée par la création d'un Centre de terminologie et d'une Commission nationale de la langue.

7. Normalisation, planification, enseignement, collecte, recherche, utilisation, etc.

8. Utilisation interne, utilisation de la part de l'administration, diffusion, utilisation pour des applications informatiques, recherche universitaire, enseignement, etc. 


\begin{tabular}{|c|c|c|}
\hline 害营 & 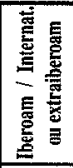 & 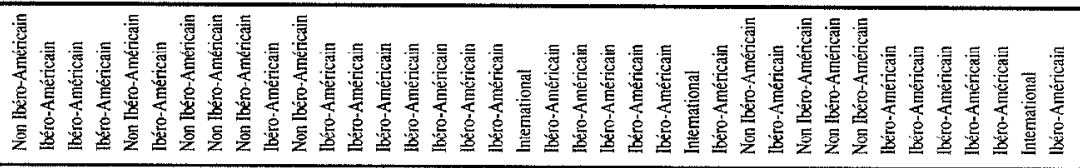 \\
\hline \multirow{2}{*}{ 象 } & 产高 & $* * * * \quad * * * * * *$ \\
\hline & 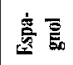 & $* * * * * * * * * * * * * *$ \\
\hline \multirow{6}{*}{ 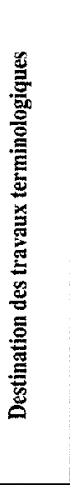 } & 言 & $x \times$ \\
\hline & 兽离 & $x \times \quad x \quad x \times x$ \\
\hline & 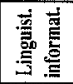 & $\times \quad \times \quad \times \quad \times \quad \times \quad \times x \times x \times x$ \\
\hline & 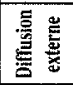 & $x x \quad x \times x \times x \quad x \quad \times \quad x \quad x$ \\
\hline & 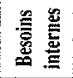 & $x \times x \times x \quad x \times x \times x \times x \times x$ \\
\hline & 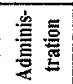 & $x \quad x$ \\
\hline \multirow{6}{*}{ 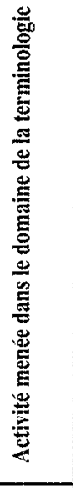 } & 言 & $x$ \\
\hline & 妾高 & $x \times x \times x \times x \quad x \quad x$ \\
\hline & 离 & $x \times \quad x \quad x \times x \times x \times x \times x$ \\
\hline & $\frac{\tilde{\Xi}}{\bar{\Xi}}$ & $x \times x \times x \times x \quad x \times \quad x \times x \times x \times x \quad x \times$ \\
\hline & 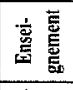 & $x \quad x$ \\
\hline & 蒿 & $x \times x$ \\
\hline \multirow{3}{*}{ 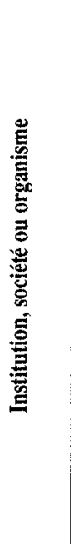 } & : & 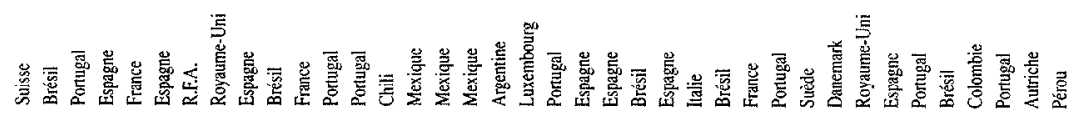 \\
\hline & $\stackrel{ \pm}{5}$ & 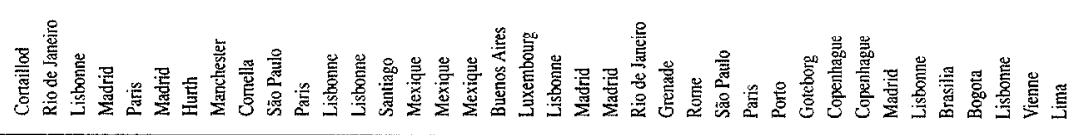 \\
\hline & 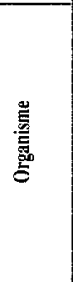 & 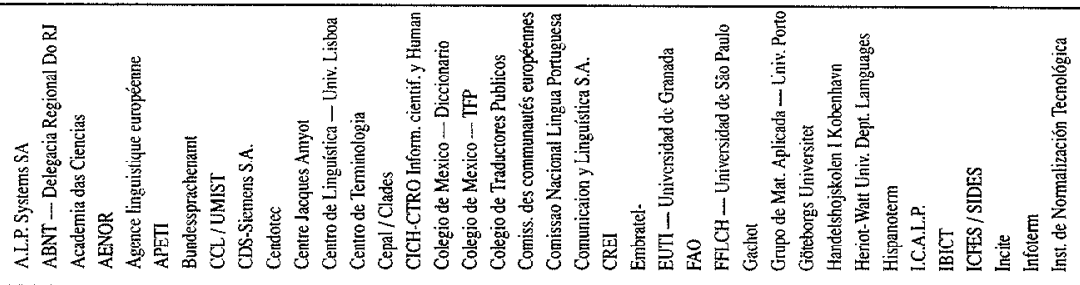 \\
\hline
\end{tabular}




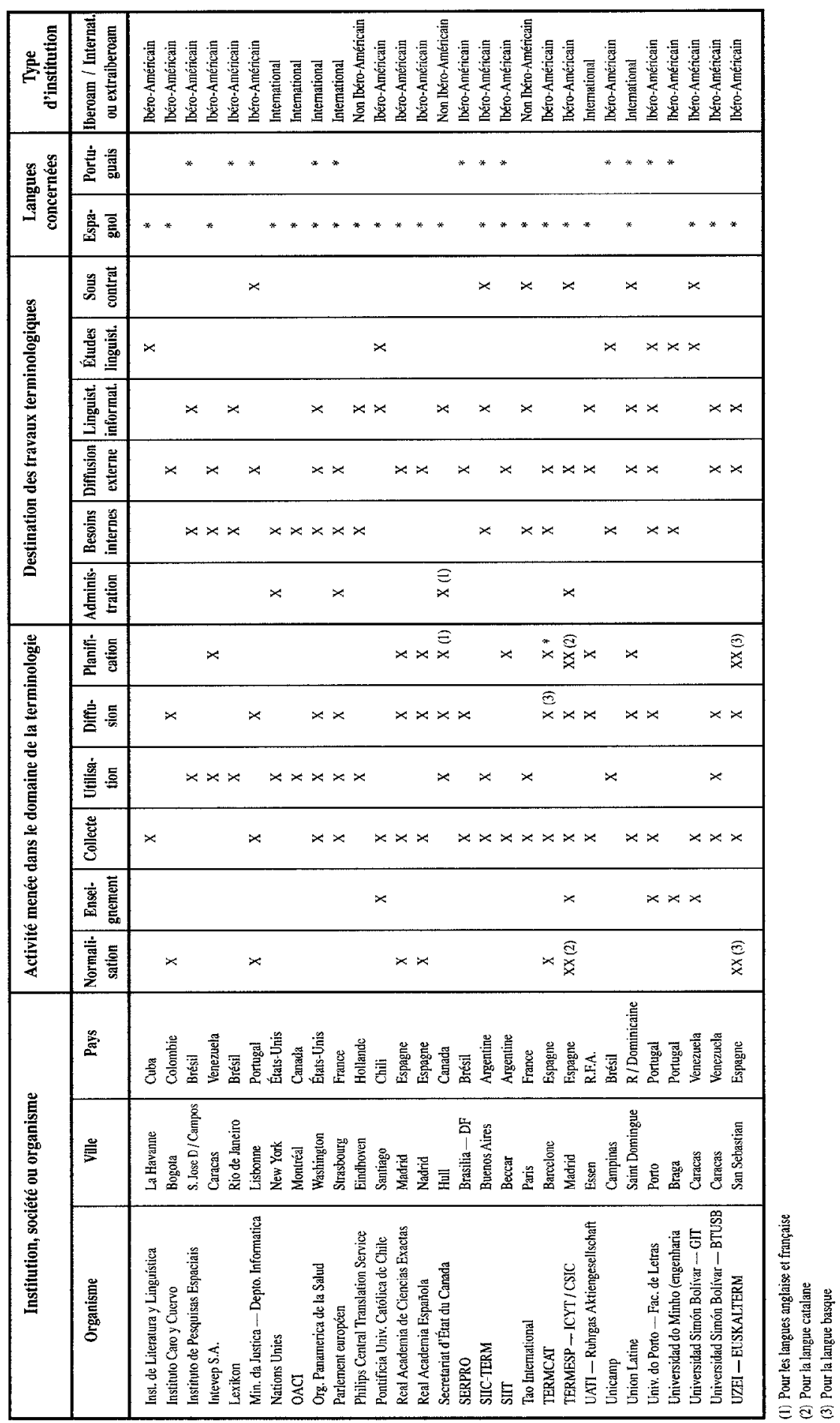

